\title{
EGFR Protein Translocation
}

National Cancer Institute

\section{Source}

National Cancer Institute. EGFR Protein Translocation. NCI Thesaurus. Code C129572.

A molecular abnormality indicating that the epidermal growth factor receptor protein has been localized to the membrane of an internal cellular compartment. 